\title{
IRF family proteins and type I interferon induction in dendritic cells
}

\author{
Prafullakumar Tailor ${ }^{1}$, Tomohiko Tamura ${ }^{1}$, Keiko Ozato ${ }^{1}$ \\ ${ }^{1}$ Laboratory of Molecular Growth Regulation, National Institute of Child Health and Human Development, National Institutes of \\ Health, Bethesda, MD 20892, USA
}

Dendritic cells (DC), although a minor population in hematopoietic cells, produce type I interferons (IFN) and other cytokines and are essential for innate immunity. They are also potent antigen presenters and regulate adaptive immunity. Among DC subtypes plasmacytoid DC (pDC) produce the highest amounts of type I IFN. In addition, pro- and anti-inflammatory cytokines such as IL-12 and IL-10 are induced in DC in response to Toll like receptor (TLR) signaling and upon viral infection. Proteins in the IRF family control many aspects of DC activity. IRF-8 and IRF-4 are essential for DC development. They differentially control the development of four DC subsets. IRF- $8^{-/-}$mice are largely devoid of pDC and CD8 $\alpha^{+}$DC, while IRF-4 ${ }^{-/}$mice lack CD4 $4^{+}$DC. IRF-8 ${ }^{-/}$, IRF4 ${ }^{-/}$, double knock-out mice have only few CD8á ${ }^{-C D} 4^{-}$DC that lack MHC II. IRF proteins also control type I IFN induction in DC. IRF-7, activated upon TLR signaling is required for IFN induction not only in pDC, but also in conventional DC (cDC) and non-DC cell types. IRF-3, although contributes to IFN induction in fibroblasts, is dispensable in IFN induction in DC. Our recent evidence reveals that type I IFN induction in DC is critically dependent on IRF-8, which acts in the feedback phase of IFN gene induction in DC. Type I IFN induction in pDC is mediated by MyD88 dependent signaling pathway, and differs from pathways employed in other cells, which mostly rely on TLR3 and RIG-I family proteins. Other pro-inflammatory cytokines are produced in an IRF-5 dependent manner. However, IRF-5 is not required for IFN induction, suggesting the presence of separate mechanisms for induction of type I IFN and other pro-inflammatory cytokines. IFN and other cytokines produced by activated DC in turn advance DC maturation and change the phenotype and function of DC. These processes are also likely to be governed by IRF family proteins.

Cell Research (2006) 16:134-140. doi:10.1038/sj.cr.7310018; published online 13 February 2006

Keywords: dendritic cells, IRF-3, 4, 5, 7, 8, type I interferon induction, activate transcription, signaling pathway dependence

\section{DC subset development specified by IRF-8 and IRF-4}

DCs are a heterogeneous population of cells with diverse functions. Based on cell surface markers, mouse splenic DCs are classified into four subtypes, the $\mathrm{B} 220^{+} \mathrm{pDC}$, $\mathrm{CD} 8 \alpha^{+} \mathrm{DC}, \mathrm{CD}^{+}{ }^{\mathrm{DC}}$ and double negative (DN) DC [1].

Correspondence: Keiko Ozato

Laboratory of Molecular Growth Regulation, NICHD, Building 6, Room 2A10, National Institutes of Health, 6 Center Drive, Bethesda MD 208922753

Tel: 301-496-9184; Fax: 301- 402-2974;

E-mail:ozatok@nih.gov

Abbreviations: DC (dendritic cells); pDC (plasmacytoid DC); cDC (conventional DC); IFN (interferons); IRF (Interferon regulatory factor); NDV (New Castle Disease virus); KO (knock out); TLR (Toll like receptor).
These cells express different sets of genes and assume distinct functions [2]. pDC produce the highest amounts of type I IFN, whereas DC $8 \alpha^{+}$DC are a major producer of IL-12p40. Other DC subsets take part in producing other pro-inflammatory as well as anti-inflammatory cytokines including IL-10 and efficiently present antigens to T cells. Despite much progress have been made on studying DC biology, the origin and pathways that direct DC development are still unclear, and it appears that DC can be generated from different cell types through multiple pathways: for example, both common lymphoid precursors and common myeloid precursors are shown to be capable of generating similar DC subsets [3]. IRF-8 and IRF-4 share a number of common features. They are expressed only in the cells of the immune system. They both interact with PU.1, an immune cell specific protein of the Ets family and regulate genes 
carrying IRF/Ets and Ets/IRF composite elements [4, 5].

We and other laboratories have recently shown that IRF-8 and IRF-4 are differentially expressed in DC subsets [6-10]. CD8 $\alpha^{+}$DC and pDC express high levels of IRF-8, but low to no IRF-4. Conversely, IRF-4 expression is high in $\mathrm{CD}^{+} \mathrm{DC}$ and DN DC where IRF-8 expression is low. Analysis of IRF-8/IRF-4 single knock-out (KO) and double knock-out (DKO) mice revealed that the above pattern of IRF-8/IRF-4 expression correlates with their requirement for DC subtype development. Thus, $\mathrm{CD} 8 \alpha^{+} \mathrm{DC}$ and $\mathrm{pDCs}$ are largely absent in IRF-8 KO mice. On the other hand, $\mathrm{CD} 4{ }^{+} \mathrm{DC}$ are missing in IRF-4 KO mice. Accordingly, DKO mice have only few DN DC, totally lacking other DC subtypes. Illustrating a profound deficiency in DC functions, DC in DKO mice are devoid of MHC class II expression. We showed that IRF-8 gene transfer rescues the development of $\mathrm{pDC}$ in vitro. As a result, type I IFN is induced in the IRF-8 transferred DC. However, IRF-4 gene transfer does not rescue IFN induction, pointing to a selective requirement of IRF-8 for IFN induction $[6,11]$. Similarly, transfer of IRF-8, but not IRF-4 rescues the development of CD $8 \alpha^{+} \mathrm{DC}$ and induction of IL-12p40 in vitro $[6,7]$. On the other hand introduction of IRF-4 restores expression of some genes selectively expressed in $\mathrm{CD}^{+} \mathrm{DC}$ [6].

The identity of progenitor cells where IRF- 8 and IRF-4 act in DC development is unknown. Nor is the mechanism by which the two factors direct DC development clear. However, observations made on the development of DC in vitro by two culture systems raise intriguing possibilities. GM-CSF and Flt3L have been used to generate different DC subsets [12]. We found that DC that develop in the presence of Flt3L preferentially depend on IRF-8, while those generated in the presence of GM-CSF rely on IRF-4 [6]. Based on these findings, a model outlined in Figure 1 may be put forward, that provides potential mechanisms and pathways by which IRF-8 and IRF-4 act. IRF-8 expression in DC progenitors may be directed by Stat 3 that is activated by Flt3L. On the other hand IRF-4 induction may be led by NF- $\kappa \mathrm{B}$ that is activated by GM-CSF. Consistent with this model, studies of conditional Stat $3 \mathrm{KO}$ mice show that Stat 3 is activated by Flt3 $\mathrm{L}$ in lineage negative bone marrow progenitor cells and Stat 3 is required for the development of pDC and $\mathrm{CD} 8 \alpha^{+} \mathrm{DC}$ [13]. The close resemblance of DC phenotypes in Stat $3 \mathrm{KO}$ mice and those of IRF-8 KO mice suggest that IRF-8 may be a downstream target of Flt3L/ Stat3 pathway [6]. GM-CSF generally stimulates "myeloid DC" (CD4 ${ }^{+}$DC and DN DC) but not $\mathrm{pDC}$ or $\mathrm{CD} 8 \alpha^{+} \mathrm{DC}$. A recent publication reports that NF- $\kappa B$ among other factors is activated by GM-CSF, which then stimulates IRF-4 expression [14]. Consistent with this report, previous studies have shown that RelB, like IRF-4, is expressed in myeloid $\mathrm{DC}$, and is required for the development of $\mathrm{CD} 4^{+} \mathrm{DC}[2$,
$15,16]$. In addition to the above IRFs, IRF-2 is reported to be required for the development of $\mathrm{CD}^{+} \mathrm{DCs}[17,18]$. Interestingly, IRF- 2 acts by inhibiting IFN $\alpha / \beta$ suppression of DC development. Although type I IFN stimulates late stage DC maturation, if overproduced, it appear to be detrimental to DC development at early stages.

As detailed below, IRF family proteins play essential roles in type I IFN gene induction. Additionally, induction of other pro-inflammatory cytokines also depends on the action of IRF proteins. It has been shown that IL-12p40, important for IFN $\gamma$ induction in NK and T cells is dependent on IRF-8 in DC $[7,9]$. IRF-1 is also required for full IL-12p40 induction in macrophages and likely to be involved in IL-12p40 induction in DC [19, 20]. Moreover, a recent report reveals that IRF-5 critically contributes to TLR mediated induction of a series of pro-inflammatory cytokines including TNF $\alpha$, IL-6 and IL-12p40 both in DC and macrophages [21]. These authors show that IRF-5, present in the cytoplasm prior to stimulation, translocates into the nucleus upon TLR signaling and binds to the IL-12p40 promoter. This process is shown to require the interaction of IRF-5 with MyD88 and TRAF6. Although IL- 6 and TNF $\alpha$ are strongly activated by NF- $\kappa B$, IRF-5 may act through putative ISREs in their promoters. On the other hand, DC developed from IRF-5 KO mice in vitro either in the presence of GM-CSF or Flt3L are shown to be capable of inducing these cytokines [22], suggesting that the requirement of IRF-5 may be contingent upon various other factors. We have previously provided evidence for the contribution of IRF-8 to the induction of various proinflammatory cytokines upon TLR9 signaling in DC [23]. These reports suggest that IRF family proteins contribute to the induction of a wide range of cytokines traditionally thought to be activated primarily by NF- $\kappa \mathrm{B}$.

\section{IFN regulation of DC maturation}

Type I IFN, when secreted from DCs not only establishes an anti-viral state in themselves and other cells, but affects their own maturation. In humans, type I IFN promotes terminal maturation of $\mathrm{CD} 34^{+}$derived DC in vitro [24]. Similarly IFN stimulates differentiation of CD14 ${ }^{+}$monocytes into DC that express high levels of MHCII [25]. Type I IFN-driven maturation of monocytes is thought to be a major feature of systemic lupus erythmatosus [26].

Similarly in mice, the acquisition of mature pDC phenotype is mediated by type I IFN produced in response to TLR signaling, and IFN-induced phenotypic changes are shown to help protect DC from apoptosis [27]. Mouse pDC are also dependent on type I IFN for migration and their clustering to the marginal zone of the spleen [28]. On the other hand, when exposed to anti-inflammatory cytokines, 
such as IL-10 and TGF $\beta$, some DCs undergo transformation to become "regulatory DC" that can promote antigen specific anergy and generation of regulatory $\mathrm{T}$ cells $[29$, 30]. Although $T$ cells are thought to be a major source of IL-10 that prompts generation of "regulatory DC", DCs themselves produce IL-10 in response to TLR signaling, and which apparently contribute to the generation of regulatory DC [24]. Similar to type I IFN induction, IL-10 production is enhanced in DC by an autocrine mechanism [31]. It will be of importance to elucidate how IRF family proteins contribute to TLR mediated maturation of DC and to the generation of "regulatory DC".

\section{Type I IFN induction in DC and non DC}

Induction of type I IFN is a key feature of DC functions [32]. In addition to the establishment of host resistance, type I IFN brings about diverse consequences on innate and adaptive immunity and affects activity and maturation of DCs themselves [33]. Before DCs were identified as the primary IFN inducers, IFN induction and its mechanisms were studied only in fibroblasts and other non DC cell types. These studies showed that IFN induction follows biphasic kinetics characterized by the activity of two IRF factors, IRF-3 and IRF-7 [34]. In the first phase, IRF-3 is phosphorylated upon TLR and viral signaling, which stimulates its dimerization, nuclear translocation, and the formation of multi-protein complex that includes the co-activator CBP and $\mathrm{p} 300$. These events lead to transcriptional induction of IFN $\beta$ and IFN $\alpha 4$, among many other type I IFN genes clustered in the $\sim 500 \mathrm{~kb}$ region of a chromosome. The noncanonical IKK homologues, IKKe/i and TBK-1 are shown to be responsible for the phosphorylation of both IRF-3 and IRF-7 [35, 36]. In addition to IRF-3/7, activation of NF- $\kappa$ B by IKK $\alpha / \beta$ is an important requirement for IFN $\beta$ gene induction [37]. The early IFN produced above then activate the classic JAK-STAT pathway and ISGF3, leading to induction of IRF-7 and other IFN inducible genes. IRF-7 establishes a positive feedback loop, and stimulates the second set of IFN genes. The IRF-7 dependent, second phase IFN induction is likely to be responsible for much of IFN produced in the cells, since fibroblasts lacking IFN $\beta$, IFNAR or IRF-7 are severely deficient in IFN production $[34,38,39]$.

Detailed molecular events that underline induction of the IFN $\beta$ gene in HeLa cells have been elucidated [37, 40]. These studies show that upon stimulation IRF-3/IRF-7 and NF- $\kappa$ B along with HMG-Y(I) form an "enhanceosome" over the promoter. This is followed by ordered recruitment of histone acetylases and chromatin remodeling factors on the promoter, resulting in the acetylation of specific lysine residues in histone $\mathrm{H} 3$ and $\mathrm{H} 4$ and remodeling of a promoter

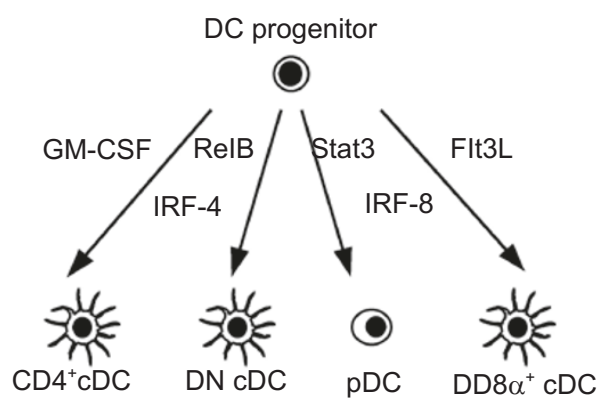

Figure 1 The IRF-8/IRF-4 paradigm in DC subset development. IRF8 and IRF-4 are differentially required for the development of four murine DC subsets. The pattern of their requirement correlates with that of their expression in DC subsets. In accord with their role in vivo DC development, IRF-8 and IRF-4 are required for in vitro DC development in the presence of Flt3L and GM-CSF, respectively. It is suggested that Stat 3 and NF- $\mathrm{KB}$ that are induced by these growth factors stimulate transcription factors that specifically activate IRF-8 and IRF-4, respectively.

proximal nucleosome. These changes highlight a major alteration in chromatin architecture and extensive modifications of histone tails in and around the gene. Previous studies showed that IRF-3 can only direct transcription of IFN $\alpha 4$ and IFN $\beta$, whereas IRF-7 can direct transcription of many IFN $\alpha$ genes in addition to the above two [34, 41]. Differential induction of IFN genes by IRF-3 and IRF-7 is attributed to the differences in their DNA binding activity and it is suggested that IRF-3 exhibits a restricted ISRE GAAANNGAAANN specificity GAAASSGAAANY in comparison to the IRF-7 consensus recognition sequence determined as GAAWNYGAAANY present in promoters of IFN genes $[42,43]$. A recent study on IRF-7 KO mice shows that viral induction of IFN $\beta$ is markedly reduced in fibroblasts lacking IRF-7, a reduction greater than in cells of IRF-3 KO mice. The reduction is seen even in an early stage of IFN induction, in line with the notion that IRF-7 acts in the first stage of IFN induction in some non DC, and play a dominant role over IRF-3 in some cases [39].

A number of papers have recently been published regarding the mechanism of type I IFN gene induction in DC (Figure 2). These studies revealed that there are significant differences between the ways in which type I IFN is induced in DCs, particularly pDC and other cells. pDC constitutively express high levels of IRF-7 and may be better equipped to respond to both the first TLR signals as well as second IFN signals than other cells and may not rely on IRF-3 as much as other cells do [44]. Indeed, pDC from IRF-7 KO mice are almost completely devoid of IFN $\alpha$ and IFN $\beta$ induction in response to viral infection and stimulation by TLR7 and 9 ligands [39]. However, 


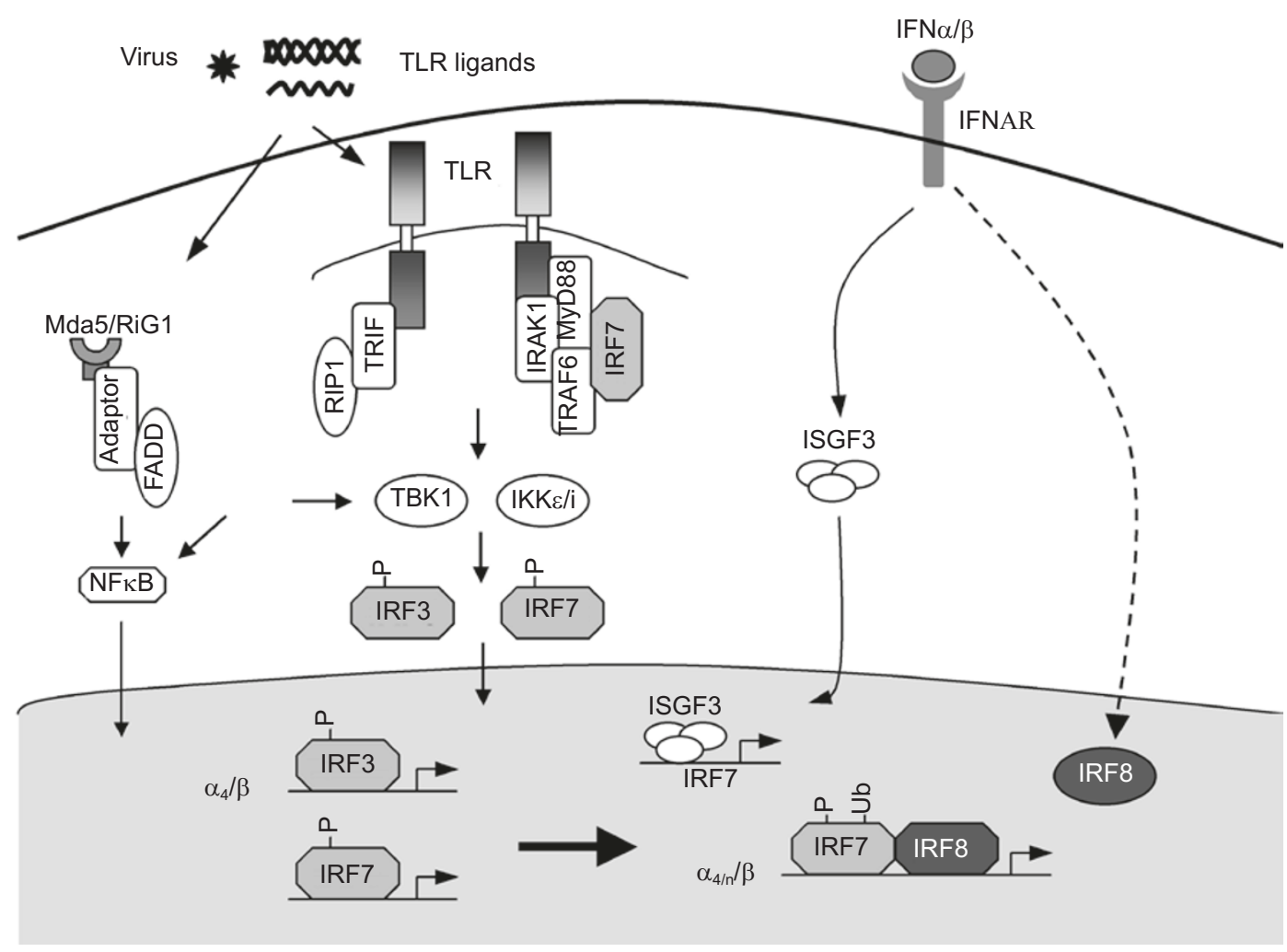

Figure 2 A diagram of type I IFN induction in pDC and cDC. In pDC, binding of ligands to TLR 7 and TLR9 activates the first phase of IFN induction mediated primarily by IRF-7 in a MyD88 dependent manner. This process involves phosphorylation and ubiquitination of IRF-7 by TBK-1/IKKe/i and TRAF6, respectively. The early IFN thus produced creates a positive feedback response to generate a second phase of IFN transcription where a much larger amount of mRNA and proteins are induced. Evidence indicates that in addition to IRF-7, IRF-8 plays a critical role in this phase of transcription. In pDC, TLR3 is absent and the RIG-1/IRF-3 pathway of IFN induction is not active. However, TLR3 and the RIG-1 pathways of IFN induction play a significant role in cDC and non DC cell types, that activate IRF-3/7 and NF- $\kappa$ B. Binding of IRF proteins to IFN promoters likely sets off a series of changes in chromatin structure and histone modification in the entire IFN gene cluster.

IFN induction in IRF-3 ${ }^{-/-}$pDC by these stimuli are comparable to that of wild type $\mathrm{pDC}$, indicating that IRF-3 is not an obligatory activator of IFN genes. Assuming that IRF-7 is the primary activator of IFN induction in pDC, it is possible that non $\alpha 4$ IFN are induced in the first phase of induction and serve as early IFN that trigger the second phase of IFN induction in a manner unique to $\mathrm{pDC}$. This possibility is supported by the report that fibroblasts from IFN $\beta \mathrm{KO}$ mice are defective in IFN production, but not splenocytes, which contain pDC [38]. Nevertheless, IFN induction in pDC also owes to the positive feedback mechanism of IFN transcription that is driven by the first phase of IFN induction, as evidenced by the abolition of IFN induction in DC derived from IFNAR KO and IRF-9 KO mice $[39,45]$.

A recent study indicates that in $\mathrm{pDC}$, IRF-7 forms a complex with MyD88, an adaptor molecule for TLR7, 8 and 9 and interacts with TRAF6, another adaptor with an E3 ligase activity that acts downstream of TLR signaling [46, 47]. As a result, IRF-7 is ubiquitinated upon TLR signaling [46]. These authors present evidence that ubiquitination of IRF-7 enhances IFN gene induction. Another interesting finding reported by Honda et al is that $\mathrm{CpG}$ is retained in the endosomal vesicles in pDC together with the MyD88-IRF7 complex for a long period of time, leading to sustained signaling and IFN $\alpha$ transcription [47]. The prolonged CpG localization in endosomal vesicles was not observed in cDC where $\mathrm{CpG}$ was rapidly delivered to lysosomes [47]. Together, IFN induction in pDC is dependent on MyD88 and the downstream signaling cascade.

Although pDC are regarded as the prime type I IFN producers, recent studies show that $\mathrm{CDC}$ also produce significant amounts of type I IFN upon infection by some viruses $[48,49]$. In contrast to the complete dependence on the MyD88-IRF-7 pathway in pDC, type I IFN induction in cDC is largely independent of MyD88 [49]. A new 
class of TLR3 independent intracellular sensor for dsRNA, RIG-1 and Mda5 has been identified [50, 51]. RIG-1 and Mda5 contain the $\mathrm{N}$-terminal caspase recruitment domain (CARD) and a C-terminal RNA helicase domain. The former domain is involved in the activation of downstream signaling, whereas the latter is responsible for recognition of dsRNA [52]. Moreover, a downstream adaptor molecule for RIG-1 and Mda5 was recently identified by four groups independently named IPS-1, MAVS, VISA and Cardif by each group [53-56]. Through different approaches these groups came to essentially the same conclusion that this adaptor is involved in the activation of NF- $\kappa B$ and IRF$3 / 7$ in a RIG-1 dependent manner. Furthermore, extension of similar studies in DC subsets showed that the RIG-1 pathway is important for viral induction of IFN in $\mathrm{cDC}$, but this pathway is dispensable in pDC [49].

pDC express TLR7 and TLR9 and ligand binding to these TLRs stimulate IFN gene transcription in a MyD88 dependent manner. However, pDC lack TLR3 and respond poorly, if at all to poly-IC, synthetic ds RNA. Also the fact that RIG-1 pathway is not active in $\mathrm{pDC}$ may raise a possibility that $\mathrm{pDC}$ have an alternative mechanism of recognizing viral dsRNA. Or it is possible that $\mathrm{pDC}$ induce IFN by recognizing viral components other than dsRNA.

In addition, FADD and RIP-1 are reported to be essential for intracellular dsRNA and virus stimulated type I interferon production $[57,58]$. Although the exact role of FADD in IFN induction is yet to be studied, FADD $^{-/}$fibroblasts are defective in type I IFN induction that is independent of IFN signaling [57]. A20, a protein originally identified as a TNF inducible protein that negatively regulates NF- $\mathrm{KB}$ activity has recently shown to interact with TBK-1 and IKK $\varepsilon$ and functions as a negative regulator of TLR3 as well, as shown by the inhibition of IFN $\beta$ promoter activity following overexpression of A20 [59]. In light of the fact that $\mathrm{A} 20$ has a deubiquitinating activity and there are several A20 like proteins carrying a similar ubiquitin-editing domain, these proteins may have a significant role in type I IFN induction in DC as well.

\section{The role of IRF-8 in DC specific type I IFN induc- tion}

Both splenic and bone marrow derived DC from IRF$8 \mathrm{KO}$ mice produce little type I IFN in response to virus infection and TLR signals, the defect of which is corrected by IRF- 8 reintroduction $[6,8,11]$. Because IRF- 8 is required for $\mathrm{pDC}$ development, these results have been taken to suggest that IRF-8 acts only during development and it has no direct role in type I IFN induction in pDC and cDC. Nevertheless, the possibility that IRF- 8 plays a significant role in directing transcription of IFN genes in
$\mathrm{pDC}$ and $\mathrm{cDC}$ has remained viable, due to the fact that IRF-8 is constitutively expressed at high levels in $\mathrm{pDC}$ and some cDC [6]. Moreover, the report that ectopic expression of IRF- 8 enhances IFN $\alpha$ and IFN $\beta$ expression further supports the idea that IRF-8 is capable of stimulating type I IFN transcription [60]. Our recent investigation provides additional evidence consistent with the active involvement of IRF-8 in IFN induction in DC. We undertook a detailed kinetic analysis of quantitative type I IFN mRNA induction upon infection with New Castle Disease virus (NDV) in bone marrow derived $\mathrm{pDC}$ and $\mathrm{cDC}$ (Tailor $\mathrm{P}$ et al., unpublished). These studies revealed that IFN transcripts induction follows biphasic kinetics that generates two distinct IFN mRNA peaks. The first peak is seen immediately after virus infection, which is followed by the generation of the second major peak at about 7 hour after infection. This is followed by a precipitous fall in IFN mRNA levels within $12 \mathrm{~h}$, indicating that IFN gene activation is coupled with a subsequent negative transcriptional regulation. We found that the second major peak was completely absent in IRF-8 ${ }^{-/}$DC, although IRF-8 ${ }^{-/}$DC produced the first peak of IFN transcripts in a manner comparable to wild type DC. The second peak most likely represents an IFN feed back response, since it coincides with the induction of IRF-7. Supporting this view, the second peak was absent in IFNAR $^{-/}$DC after NDV infection, consistent with previous reports $[39,45]$. These results are in line with the view that IRF-8 takes part in the feedback phase of IFN transcription, although it is not required for the initial phase of IFN induction. In accordance with this view, we found that IRF-8 bound to the IFN gene promoters and that nuclear translocation of IRF-3 and IRF-7 as well as induction of IRF-7 after NDV infection occurred normally in IRF- ${ }^{-/-}$DC. The participation of IRF-8 would ensure quantitative amplification of IFN transcription beyond that achieved by IRF-7 alone. Together, through the participation of IRF-8, type I IFN induction in DC appears to bear an additional unique feature that extends from signaling to the transcriptional mechanism itself. Further, the fact that IRF-8 can exert a dual activity in transcription $[4,5]$ may suggest that it plays a role in orchestrating post-activation events taking place in the chromatin of IFN gene cluster in DC.

\section{Conclusions and Perspectives}

DCs activate IFN transcription by employing a number of unique mechanisms that ensure immediate production of type I IFN in large quantity. The early IFN production helps to confer effective innate immunity after pathogen infection prior to the establishment of adaptive immunity. It is now well established that IRF proteins, in particular IRF-7 is essential for type I IFN induction in DC. Our study 
indicates that IRF-8 also has a pivotal role in DC specific IFN transcription. Future work is needed to elucidate the mechanism by which the IRF factors are recruited to the IFN promoters and activate transcription. An additional question as to how IRF proteins modify core histones in nucleosomes and remodel chromatin in the IFN cluster also awaits future investigations.

\section{Acknowledgements}

We thank Drs L Gabriele and G Trinchieri for discussions. This work was supported in part by the Intramural Program of the NICHD and the Trans-NIH/FDA Intramural Biodefense Program of the NIAID, USA.

\section{References}

1 Carbone FR, Heath WR. The role of dendritic cell subsets in immunity to viruses. Curr Opin Immunol 2003; 15:416-20.

2 Edwards $\mathrm{AD}$, Chaussabel D, Tomlinson S, et al. Relationships among murine $\mathrm{CD} 11 \mathrm{c}$ (high) dendritic cell subsets as revealed by baseline gene expression patterns. J Immunol 2003; 171:4760.

3 Manz MG, Traver D, Miyamoto T, et al. Dendritic cell potentials of early lymphoid and myeloid progenitors. Blood 2001; 97:3333-41.

4 Tamura T, Ozato K. ICSBP/IRF-8: its regulatory roles in the development of myeloid cells. J Interferon Cytokine Res 2002; 22:145-52.

5 Levi BZ, Hashmueli S, Gleit-Kielmanowicz M, et al. ICSBP/IRF8 transactivation: a tale of protein-protein interaction. J Interferon Cytokine Res 2002; 22:153-60.

6 Tamura T, Tailor P, Yamaoka K, et al. IFN regulatory factor-4 and -8 govern dendritic cell subset development and their functional diversity. J Immunol 2005; 174:2573-81.

7 Tsujimura H, Tamura T, Gongora C, et al. ICSBP/IRF-8 retrovirus transduction rescues dendritic cell development in vitro. Blood 2003; 101:961-9.

8 Schiavoni G, Mattei F, Sestili P, et al. ICSBP Is Essential for the Development of Mouse Type I Interferon-producing Cells and for the Generation and Activation of CD8 \{alpha $\}+$ Dendritic Cells. J Exp Med 2002; 196:1415-25.

9 Aliberti J, Schulz O, Pennington DJ, et al. Essential role for ICSBP in the in vivo development of murine $\mathrm{CD} 8 \alpha^{+}$dendritic cells. Blood 2003; 101:305-10.

10 Suzuki S, Honma K, Matsuyama T, et al. Critical roles of interferon regulatory factor 4 in CD11bhighCD8 $\alpha$ lpha- dendritic cell development. Proc Natl Acad Sci U S A 2004; 101:8981-6.

11 Tsujimura H, Tamura T, Ozato K. Cutting edge: IFN consensus sequence binding protein/IFN regulatory factor 8 drives the development of type I IFN-producing plasmacytoid dendritic cells. J Immunol 2003; 170:1131-5.

12 Gilliet M, Boonstra A, Paturel C, et al. The development of murine plasmacytoid dendritic cell precursors is differentially regulated by FLT3-ligand and granulocyte/macrophage colonystimulating factor. J Exp Med 2002; 195:953-8.

13 Laouar Y, Welte T, Fu XY, Flavell RA. STAT3 is required for
Flt3L-dependent dendritic cell differentiation. Immunity 2003; 19:903-12.

14 Lehtonen A, Veckman V, Nikula T, et al. Differential expression of IFN regulatory factor 4 gene in human monocyte-derived dendritic cells and macrophages. J Immunol 2005; 175:6570-9.

15 Grumont RJ, Gerondakis S. Rel induces interferon regulatory factor 4 (IRF-4) expression in lymphocytes: modulation of interferon-regulated gene expression by rel/nuclear factor kappaB. J Exp Med 2000; 191:1281-92.

$16 \mathrm{Wu} \mathrm{L}, \mathrm{D}$ 'Amico A, Winkel KD, et al. RelB is essential for the development of myeloid-related CD8alpha- dendritic cells but not of lymphoid-related CD8alpha+ dendritic cells. Immunity 1998; 9:839-47.

17 Honda K, Mizutani T, Taniguchi T. Negative regulation of IFNalpha/beta signaling by IFN regulatory factor 2 for homeostatic development of dendritic cells. Proc Natl Acad Sci U S A 2004; 101:2416-21.

18 Ichikawa E, Hida S, Omatsu Y, et al. Defective development of splenic and epidermal CD4+ dendritic cells in mice deficient for IFN regulatory factor-2. Proc Natl Acad Sci U S A 2004; 101:3909-14.

19 Taki S, Sato T, Ogasawara K, et al. Multistage Regulation of Th1-Type Immune Responses by the Transcription Factor IRF-1. Immunity 1997; 6:673-9.

20 Lohoff M, Ferrick D, Mittrucker H-W, et al. Interferon Regulatory Factor-1 Is Required for a T Helper 1 Immune Response In Vivo. Immunity 1997; 6:681-9.

21 Takaoka A, Yanai H, Kondo S, et al. Integral role of IRF-5 in the gene induction programme activated by Toll-like receptors. Nature 2005; 434:243-9.

22 Negishi H, Ohba Y, Yanai H, et al. Negative regulation of Tolllike-receptor signaling by IRF-4. Proc Natl Acad Sci U S A 2005; 102:15989-94.

23 Tsujimura H, Tamura T, Kong HJ, et al. Toll-like receptor 9 signaling activates NF-kappaB through IFN regulatory factor$8 /$ IFN consensus sequence binding protein in dendritic cells. J Immunol 2004; 172:6820-27.

24 Luft T, Pang KC, Thomas E, et al. Type I IFNs enhance the terminal differentiation of dendritic cells. J Immunol 1998; 161:1947-53.

25 Santini SM, Di Pucchio T, Lapenta C, et al. A New Type I IFNMediated Pathway for the Rapid Differentiation of Monocytes into Highly Active Dendritic Cells. Stem Cells 2003; 21:35762.

26 Banchereau J, Pascual V, Palucka AK. Autoimmunity through cytokine-induced dendritic cell activation. Immunity 2004; 20:539-50.

27 O'Keeffe M, Hochrein H, Vremec D, et al. Mouse plasmacytoid cells: long-lived cells, heterogeneous in surface phenotype and function, that differentiate into $\mathrm{CD} 8(+)$ dendritic cells only after microbial stimulus. J Exp Med 2002; 196:1307-9.

28 Asselin-Paturel C, Trinchieri G. Production of type I interferons: plasmacytoid dendritic cells and beyond. J Exp Med 2005; 202:461-5.

29 Wakkach A, Fournier N, Brun V, et al. Characterization of dendritic cells that induce tolerance and $\mathrm{T}$ regulatory 1 cell differentiation in vivo. Immunity 2003; 18:605-7.

30 Cao X-T. cytokines required for the generation and function of regulatory dendritic cells. Cell Research 2005; 15:61. 
31 Corinti S, Albanesi C, la Sala A, et al. Regulatory Activity of Autocrine IL-10 on Dendritic Cell Functions. J Immunol 2001; 166:4312-8.

32 Dalod M, Salazar-Mather TP, Malmgaard L, et al. Interferon $\alpha / \beta$ and Interleukin 12 Responses to Viral Infections: Pathways Regulating Dendritic Cell Cytokine Expression In Vivo. J Exp Med 2002; 195:517-8.

33 Biron CA. Interferons alpha and beta as immune regulators-a new look. Immunity 2001; 14:661-4.

34 Marie I, Durbin JE, Levy DE. Differential viral induction of distinct interferon-alpha genes by positive feedback through interferon regulatory factor-7. EMBO J 1998; 17:6660-9.

35 Fitzgerald KA, McWhirter SM, Faia KL, et al. IKK $\varepsilon$ and TBK1 are essential components of the IRF3 signaling pathway. Nat Immunol 2003; 4:491-6.

36 Sharma S, tenOever BR, Grandvaux N, et al. Triggering the Interferon Antiviral Response Through an IKK-Related Pathway. Science 2003; 300:1148-51.

37 Agalioti T, Lomvardas S, Parekh B, et al. Ordered Recruitment of Chromatin Modifying and General Transcription Factors to the IFN- $\beta$ Promoter. Cell 2000; 103:667-78.

38 Erlandsson L, Blumenthal R, Eloranta M-L, et al. Interferon- $\beta$ is required for interferon- $\alpha$ production in mouse fibroblasts. Current Biology 1998; 8:223-6.

39 Honda K, Yanai H, Negishi H, et al. IRF-7 is the master regulator of type-I interferon-dependent immune responses. Nature 2005; 434:772-7.

40 Munshi N, Agalioti T, Lomvardas S, et al. Coordination of a Transcriptional Switch by HMGI(Y) Acetylation. Science 2001; 293:1133-6.

41 Sato M, Suemori H, Hata N, et al. Distinct and Essential Roles of Transcription Factors IRF-3 and IRF-7 in Response to Viruses for IFN- $\alpha / \beta$ Gene Induction. Immunity 2000; 13:539-48.

42 Lin R, Genin P, Mamane Y, Hiscott J. Selective DNA Binding and Association with the CREB Binding Protein Coactivator Contribute to Differential Activation of Alpha/Beta Interferon Genes by Interferon Regulatory Factors 3 and 7. Mol Cell Biol 2000; 20:6342-53.

43 Civas A, Island ML, Genin P, et al. Regulation of virus-induced interferon-A genes. Biochimie 2002; 84:643-54.

44 Dai J, Megjugorac NJ, Amrute SB, Fitzgerald-Bocarsly P. Regulation of IFN regulatory factor-7 and IFN-alpha production by enveloped virus and lipopolysaccharide in human plasmacytoid dendritic cells. J Immunol 2004; 173:1535-48.

45 Gautier G, Humbert M, Deauvieau F, et al. A type I interferon autocrine-paracrine loop is involved in Toll-like receptor-induced interleukin-12p70 secretion by dendritic cells. J Exp Med 2005;
201:1435-46.

46 Kawai T, Sato S, Ishii KJ, et al. Interferon-alpha induction through Toll-like receptors involves a direct interaction of IRF7 with MyD88 and TRAF6. Nat Immunol 2004; 5:1061-8.

47 Honda K, Ohba Y, Yanai H, et al. Spatiotemporal regulation of MyD88-IRF-7 signalling for robust type-I interferon induction. Nature 2005; 434:1035-40.

48 Diebold SS, Montoya M, Unger $\mathrm{H}$, et al. Viral infection switches non-plasmacytoid dendritic cells into high interferon producers. Nature 2003; 424:324-28.

49 Kato H, Sato S, Yoneyama M, et al. Cell type-specific involvement of RIG-I in antiviral response. Immunity 2005; 23:19-28.

50 Yoneyama M, Kikuchi M, Natsukawa T, et al. The RNA helicase RIG-I has an essential function in double-stranded RNA-induced innate antiviral responses. Nat Immunol 2004; 5:730-7.

51 Kang DC, Gopalkrishnan RV, Wu Q, et al. mda-5: An interferoninducible putative RNA helicase with double-stranded RNAdependent ATPase activity and melanoma growth-suppressive properties. Proc Natl Acad Sci U S A 2002; 99:637-42.

52 Yoneyama M, Kikuchi M, Matsumoto K, et al. Shared and unique functions of the DExD/H-box helicases RIG-I, MDA5, and LGP2 in antiviral innate immunity. J Immunol 2005; 175:2851-8.

53 Kawai T, Takahashi K, Sato S, et al. IPS-1, an adaptor triggering RIG-I- and Mda5-mediated type I interferon induction. Nat Immunol 2005; 6:981-8.

54 Seth RB, Sun L, Ea CK, Chen ZJ. Identification and characterization of MAVS, a mitochondrial antiviral signaling protein that activates NF-kappaB and IRF 3. Cell 2005; 122:669-82.

55 Xu LG, Wang YY, Han KJ, et al. VISA Is an Adapter Protein Required for Virus-Triggered IFN- $\beta$ Signaling. Molecular Cell 2005; 19:727-40.

56 Meylan E, Curran J, Hofmann K, et al. Cardif is an adaptor protein in the RIG-I antiviral pathway and is targeted by hepatitis C virus. Nature 2005; 437:1167-72.

57 Balachandran S, Thomas E, Barber GN. A FADD-dependent innate immune mechanism in mammalian cells. Nature 2004; 432:401-5.

58 Meylan E, Burns K, Hofmann K, et al. RIP1 is an essential mediator of Toll-like receptor 3-induced NF-kappa B activation. Nat Immunol 2004; 5:503-7.

59 Saitoh T, Yamamoto M, Miyagishi M, et al. A20 is a negative regulator of IFN regulatory factor 3 signaling. J Immunol 2005; 174:1507-12.

60 Nardi V, Mohammad A, Dikovskaia E, Daley GQ. Interferons and IRF8/ICSBP Stimulate Immune Protection Against BCR/ABLInduced Leukemia. Blood (ASH Annual Meeting Abstracts) 2004; 104:2965. 